\title{
LENGTH SCALES FOR THE FRACTURE OF NANOSTRUCTURES
}

\author{
William W. Gerberich ${ }^{\dagger}$ John Jungk $^{\dagger}$ and Alex A. Volinsky ${ }^{\star}$ \\ †Department of Chemical Engineering and Materials Science \\ University of Minnesota, Minneapolis, MN 55455 \\ Motorola Corp., Mesa AZ 85202
}

\begin{abstract}
Length scales are essential to the understanding of small volume deformation and fracture in emerging technologies. Recent analysis by two groups at the atomistic [1] and mesoscopic [2] levels have shown the importance of the volume to surface ratio to the indentation size effect (ISE) at small depths of penetration. We have interpreted this in terms of the plastic work under the contact and the surface work associated with the creation of new surface or the excess surface stress. Treating this as a modified Griffith criterion the case is made that this same length scale should apply to the delamination of thin films. By making this simple equivalency in length scales, an $R$-curve analysis for crack growth resistance, $G_{R}$, in thin film delamination emerges. This recovers the classic $\sigma_{y s}^{2} h / E$ term as well as the fact that interfacial toughness should scale with the square root of incremental crack growth. Here $\sigma_{y s}$ is yield strength, $h$ is thickness and $E$ is modulus of the film. As applied to thin $\mathrm{Cu}$ films bonded to silicon substrates with a thin innerlayer of Ti, the model is in good agreement.
\end{abstract}

\section{KEY WORDS}

Nanoscale lengths, thin films, delamination energy

\section{INTRODUCTION}

Current research into nanotechnology is increasingly aware of the limitations of small scales in micromachines, MEMS, microelectric interconnects and magnetic recording heads. One of these limitations is when device reliability is compromised by poor thin film adhesion. The last decade has seen a considerable effort at applying linear elastic fracture mechanics concepts toward film fracture problems [3-5]. To a lesser extent has there been progress in understanding the elasticplastic thin-film delamination problem particularly on the experimental side of the ledger. This is partly because of a host of possible length scale variables that can be appropriately incorporated to fit any given set of data. The confusion comes in as to how to exactly measure an appropriate length scale and as to which one(s) should be included. To illustrate the problem, we enumerate in 
Table 1 some of the possible length scales and evolutionary microstructural features of importance to small volume deformation and fracture. The first five parameters with asterisks are some of the possible fundamental length scales which should be considered in thin film deformation and fracture. We consider interplanar slip band spacing because of possible sub-cell structures which might evolve during a thermal-mechanical processing history. The remaining parameters can easily evolve during the deformation and fracture history, e.g. those associated with nanoindentation induced yield or fracture events.

TABLE 1

\section{POSSIBLE LENGTH SCALES* AND EVOLUTIONARY MICROSTRUCTURAL FEATURES OF IMPORTANCE}

IN THE DEFORMATION AND FRACTURE OF SMALL VOLUMES

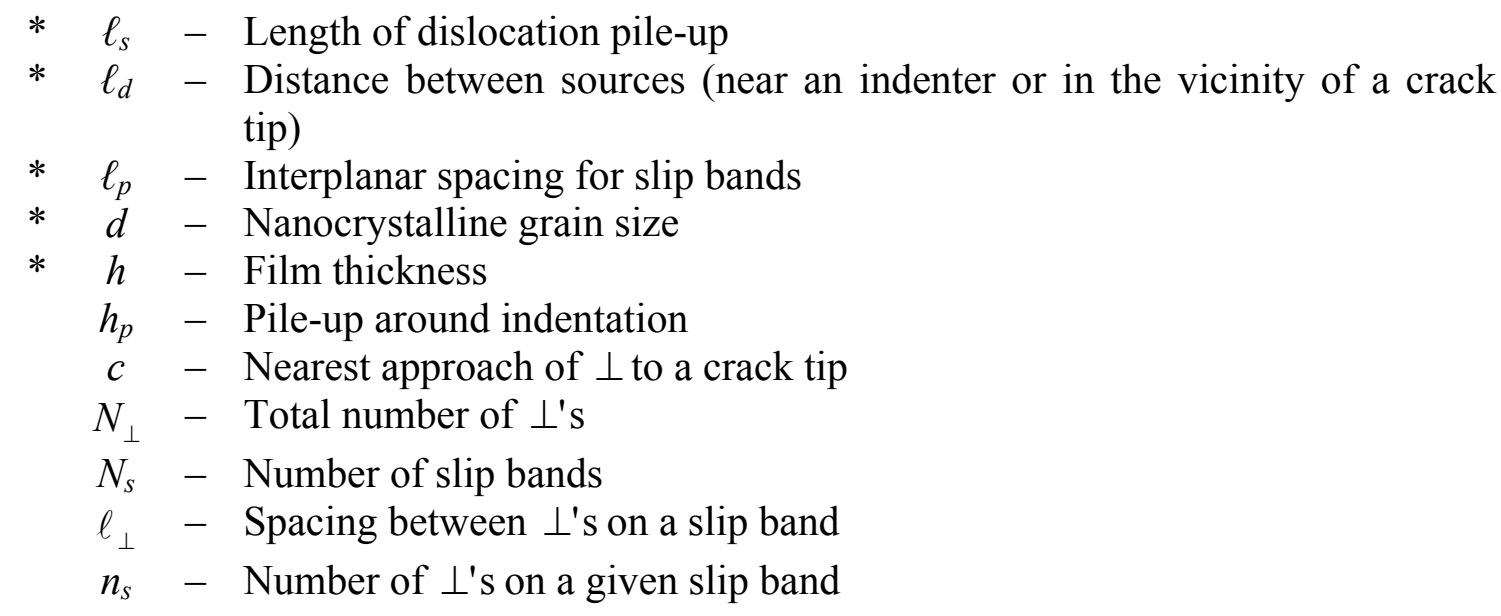

We believe we have fortuitously arrived at a single length scale parameter that controls both small volume deformation and fracture behavior of thin films.

This occurred because of two separate pathways we have been following, one dealing with the indentation size effect (ISE) in bulk crystals and one addressing delamination fracture resistance of thin films. While these seem quite disparate their commonality was that both phenomena were studied by nanoindentation. These included the ISE associated with an abrupt yield excursion in single crystals [2,6] and interfacial fracture resistance from indentation induced blister formation $[7,8,16]$, both of which involved small volume deformation and fracture. For a number of years now we have been drawing a parallel between the point forces associated with a crack tip and an indenter tip [9] and the fact that the driving forces for the two might evolve similar localized dislocation arrangements. In that paper [9] we also noted that the pile-up height around an indentation behaves similarly, as a function of load, for both continuum and discretized models. This further suggested that contact mechanics might provide the connective link between mesoscopic and continuum models. However, it wasn't until we examined the same thin film system using these two approaches that we could convince ourselves that this is truly the case and that the connective link is the length scale that controls deformation and fracture of small volumes. What we have been able to show is that with no unknown constants or parameters, the deformation length scale directly determined from a series of nanoindentations into thin films leads to an $R$ curve analysis for the delamination fracture resistance of those same films. This is currently shown for two $\mathrm{Cu}$ films of $120 \mathrm{~nm}$ and $3.3 \mu \mathrm{m}$ thickness bonded with Ti to silicon wafers. 


\section{THEORETICAL BACKGROUND}

Here we consider in order some theoretical background for length scales as applied to the deformation of bulk material and the deformation and delamination of thin films, all at small scale. Specifically, we address the deformation and fracture response to nanoindentation at the nanometer scale.

\section{Length Scale(s) for Bulk Deformation}

Consider a conical tip with a $70 \mathrm{~nm}$ tip radius indenting into a surface producing concentric dislocation loops along glide cylinders. When an oxide breakthrough event occurs these travel back to the free surface resulting in pile-up as schematically shown in Figure 1. The sketched semicircle is the calculated "elastic-plastic" boundary from continuum theory. From experimental observations of dislocation rosette patterns, we know that dislocations extend well beyond the continuum estimate. For example, from AFM profiles, we know the rosette pattern extended well beyond the pile-up region observed at the surface, measured to be $500 \mathrm{~nm}$ and $>3000 \mathrm{~nm}$, respectively [10]. In addition, we now know that dislocations are emitted under the tip prior to the displacement excursion and that these are released commensurate with the yield excursion. Nevertheless, the continuum theory appears to capture the essence of the plastic pile-up process.

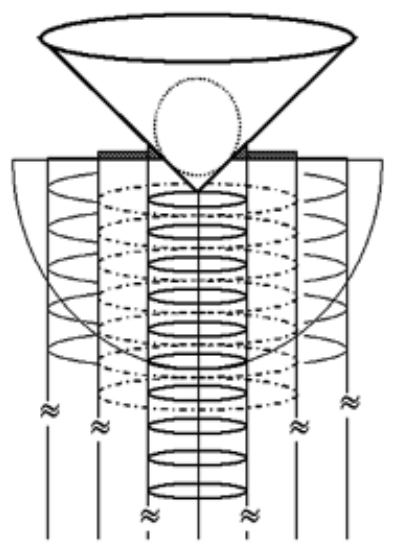

Figure 1: For the sample case of a $70 \mathrm{~nm}$ tip radius, the contact radius and plastic zone size are drawn to scale. The corresponding pile-up estimated from Eq. (1) would be $8.8 \mathrm{~nm}$ representing 35 dislocation loops.

An example of a yield excursion and the corresponding pile-ups in a $<100>$ Ta single crystal is shown in Figure 2. In a recent paper [10], the continuum representation of pile-up [11] was found to give a good fit to plastic pile-up as determined by AFM measurement. For the contact radius, $a$ being much smaller than the plastic zone size radius, $c$, plastic pile-up, $h_{p}$ at the contact edge is given by

$$
h_{p}(a) \cong(1-v) \frac{\sigma_{y s}}{E}\left(\frac{c^{3}}{a^{2}}\right) \frac{\pi}{2}
$$

as formulated from Johnson's cavity model of contact mechanics [12]. In principal, this maximum pile-up should relate to the release of dislocations forming pile-up, as is idealized in Figure 1. Given that pile-up should be some fraction of the dislocations formed during the yield excursion, $\delta_{e x c}$, and that these to first order are $\delta_{\text {exc }} / b$ dislocations, where $b$ is the Burger's vector, we find

$$
h(a) \cong \alpha^{\prime} n_{s} b \quad ; \quad \alpha^{\prime}<1
$$




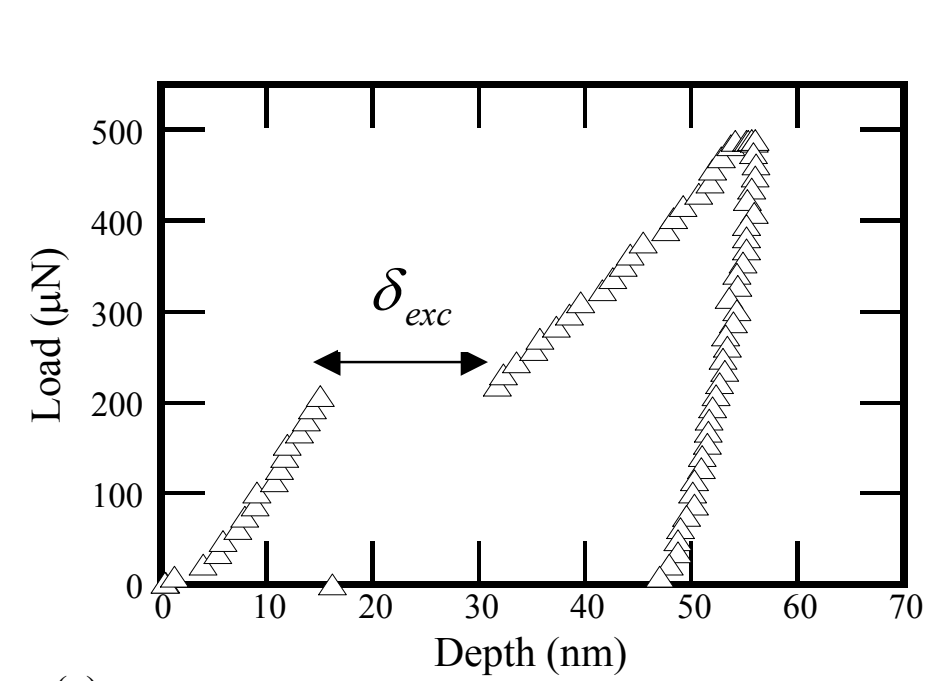

(a)

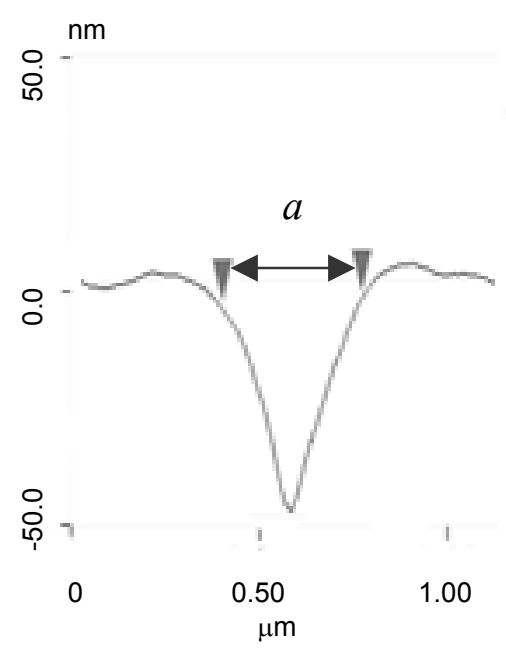

(b)

Figure 2: $\quad$ Yield excursion around a $1 \mu \mathrm{m}$ radius diamond tip showing (a) displacement jump and (b) resulting pile-up measured by AFM.

From previous works [2] on the indentation size effect (ISE) we had shown that the volume to surface ratio could explain the increases in hardnesses observed at very small depths of penetration with $\delta \leq 300 \mathrm{~nm}$. The hypothesis was that the surface work was commensurate with the volume work and that this led to a constant volume $(V)$ to surface ratio $(S)$ ratio at small depths. We had previously described this plastic volume under the tip, $2 \pi c^{2} / 3$, and contact surface area, $\pi a^{2}$, as a length scale, $\ell_{s}$, giving

$$
\ell_{s}=\frac{V}{S}=\frac{2 c^{3}}{3 a^{2}}
$$

in terms of the contact radius, $a$, and plastic zone radius, $c$. Furthermore, the volume work of plastic deformation $\tau_{y s} c^{2} \delta / 2$ and the surface work $\pi a^{2} \gamma_{s}$ gave

$$
\frac{W_{V}}{W_{S}}=\frac{\tau_{y s} \delta c^{2}}{\gamma_{s} \pi a^{2}}
$$

Combining (3) and (4) gives this work ratio in terms of the length scale to be

$$
\frac{W_{V}}{W_{S}}=\frac{3 \tau_{y s} \delta \ell_{s}}{4 \pi \gamma_{s} c}
$$

From the data presented elsewhere [6] this ratio is shown for two sets of $<100>$ single crystal data of $\mathrm{Al}$ and $\mathrm{W}$ where three different tip radii were utilized in each case. A fourth sharpest tip for each was not considered to avoid complications of nonspherical contact. These data in Figure 3 strongly suggest that the surface work is an appreciable portion of the total work for the first several hundred nanometers of penetration. The separation of the data within each material is clearly due to the $\delta / c$ ratio with sharper tips producing a greater plastic penetration for the same plastic zone size producing a greater portion of volume work. The separation of the data between the two materials is mostly due to the yield stress to surface energy ratio with the dimensionless parameter $\tau_{y s} \ell_{s} / \gamma_{s}$ being 670 for $<100>\mathrm{W}$ and 240 for $<100>\mathrm{Al}$. This further suggests that for 
small length scales the surface work could become extremely important as this dimensionless parameter drops below 100.

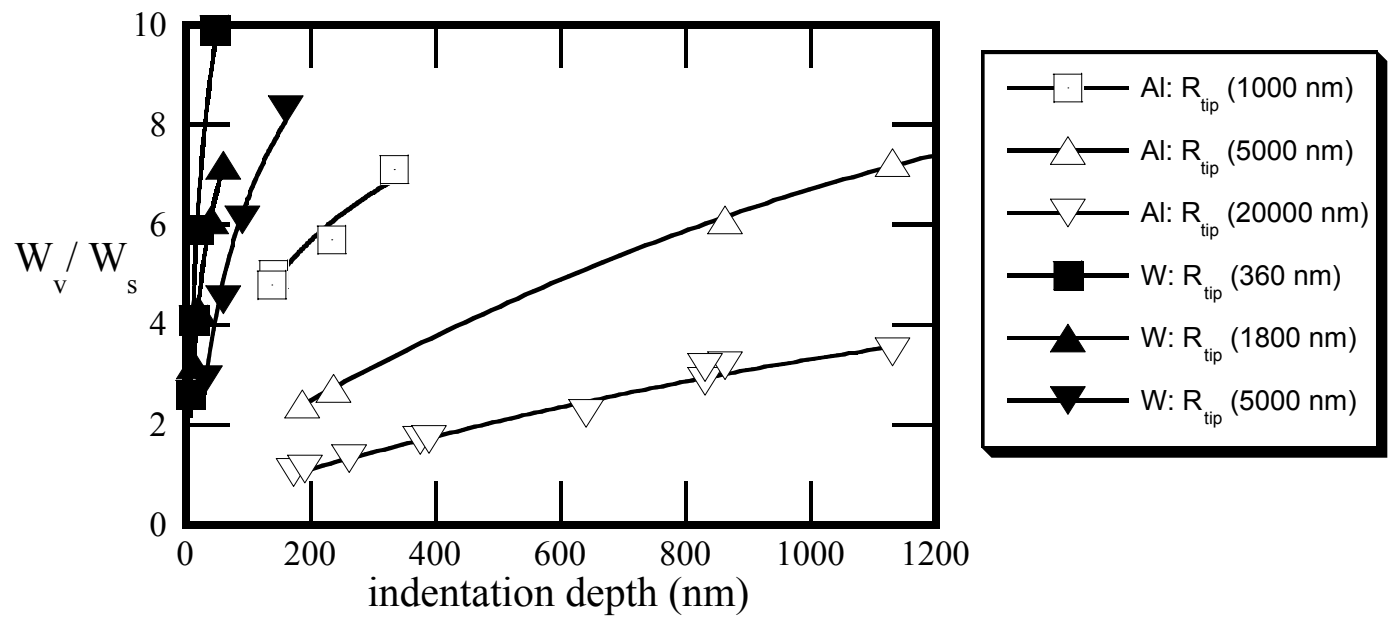

Figure 3: Volume to surface work ratio as a function of depth for a tungsten single crystal with $\ell_{s} \cong 4700 \mathrm{~nm}, \tau_{y s}=400 \mathrm{MPa}$ and $\gamma_{s}=2.8 \mathrm{~J} / \mathrm{m}^{2}$ and for an aluminum single crystal with $\ell_{s} \cong 7900 \mathrm{~nm}, \tau_{y s}=30 \mathrm{MPa}$ and $\gamma_{s}=1.0 \mathrm{~J} / \mathrm{m}^{2}$. Plastic zone sizes are those reported elsewhere [6].

This prompted us to examine Eqs. (1) and (2) more closely since the plastic pile-up of Eq. (1) is directly related to this length scale as it contains $c^{3} / a^{2}$. As we have recently discussed [10], the number of dislocations emitted, which then form a piled-up slip band, is obtained from Eqs. (1) and (2) eliminating $h(a)$ to give

$$
n_{s}=\frac{\pi(1-v) \sigma_{y s} c^{3}}{2 \alpha^{\prime} b E a^{2}}
$$

Elimination of $c^{3} / a^{2}$ through Eq. (3) and substituting $\tau_{y s} / \mu$ for $\sigma_{y s} / E$ gives

$$
n_{s}=\frac{\pi \ell_{s}(1-v)}{2 b \alpha} \frac{\tau_{y s}}{\mu}
$$

with $\alpha=\alpha^{\prime} \cdot(3 / 2)$. Since $\alpha^{\prime}<1$, it is tempting to take $\alpha \sim 1$ which then makes Eq. (7) identical to the simple pile-up theory of Eshelby et al. [13]. The simplified picture of Figure 1 is that an inverse pile-up forms and at the yield excursion these release into the free surface causing topographical pile-up around the indenter tip. It is significant that Eq. (1) is from continuum theory appropriate to the macroscale while Eq. (7) is from dislocation theory appropriate to the mesoscale.

Because this appeared to work so well for small volume penetration into bulk single crystals, it was decided to apply this to the deformation of thin metal films bonded to and constrained by relatively rigid elastic substrates. Herein then lies the key. When we drastically change the size scale of the component as in thin films, how does this change the length scale(s) appropriate to deformation and fracture. As discussed above, in Table 1 we enumerated the possible length scales and a few of the evolving structural parameters that might be involved in deformation and fracture of thin films. The length scale for a thin film, clearly smaller than that for a corresponding 
single crystal, could be a dislocation pile-up length, a nanocrystalline grain size, $d$, which scales with thickness but not necessarily linearly, or film thickness, $h$, itself. The other parameters in Table 1 tend to be evolutionary during a point contact process and can eventually lead to friction changes, wear, film fracture or film delamination. Let us first consider definition of the $V / S$ value for thin films as may be important to any of these deformation and fracture processes. This is then followed by application to a film delamination process.

\section{Length Scale for Thin Film Deformation}

An in-depth series of tests [14] for four different aluminum films of $0.34,0.5,1$ and $2 \mu \mathrm{m}$ thickness gave the relationship between the plastic zone size to indenter contact radius, $c / a$, and the normalized film thickness to be

$$
\left(\frac{c}{a}\right)^{2}=\frac{\alpha h}{a}
$$

with $\alpha=5.3$. The extent of plasticity depends on the degree of constraint and the yield stress. For smaller thicknesses, $h$, the substrate more easily constrains plastic flow coupling with an elevated yield stress to reduce $c / a$. For greater penetration and hence greater contact radii, $a$, the constraint factor reduces $c / a$. For a thin film, it is simple to translate this into a volume to surface ratio for indenters of rotational symmetry, cylinders, spheres or cones. A contact radius, $a$, giving a surface area of $\pi a^{2}$ and a constrained plastic zone, $c$, of $\pi c^{2} h$ gives

$$
\ell_{s}=\left.\frac{V}{S}\right|_{\text {constrained }}=\frac{\pi c^{2} h}{\pi a^{2}} .
$$

See the schematic of Figure 4(a).

Elimination of $c$ through (8) and (9) gives

$$
\ell_{s}=\frac{\alpha h^{2}}{a}
$$

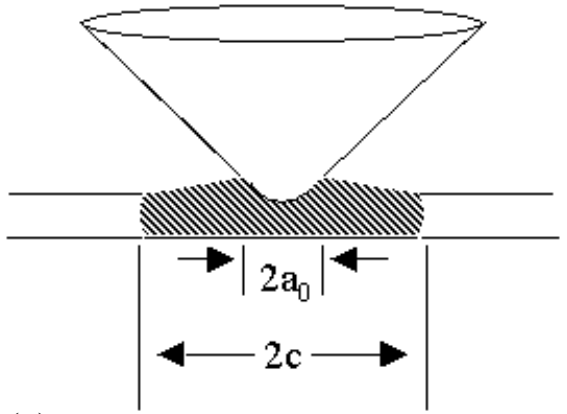

(a)

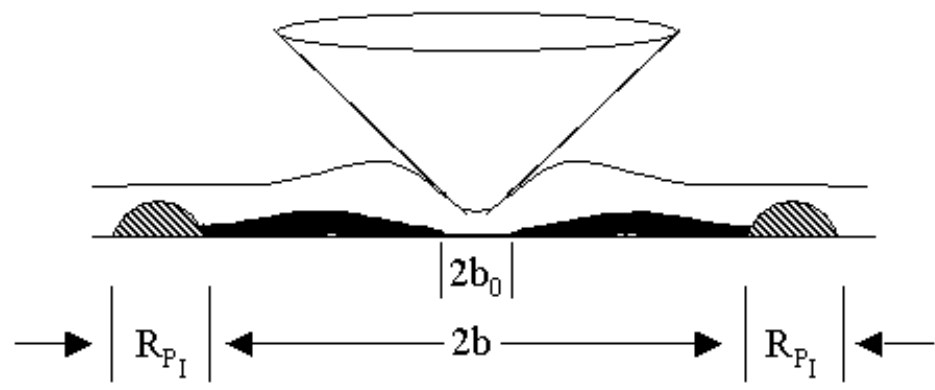

(b)

Figure 4: Plastic volumes associated with $V / S$ for (a) a conical indenter into a constrained thin film producing a plastic zone radius of $c$; (b) a conical indenter producing a contact diameter of $2 a_{0}$ producing an initial crack of $\sim 2 b_{0}$ which grows to $2 b$ under increasing load forming a plastic zone of $R_{P_{I}}$. 
In reality this appears to be a mixed length scale since it depends both on the film thickness and the sharpness of the indenter which controls the contact radius for a given penetration depth. One notes that this length scale can be quite small for film thicknesses on the order of $300 \mathrm{~nm}$. Since a $1 \mu \mathrm{m}$ radius indenter penetrating to the film/substrate interface would give a contact radius of 720 $\mathrm{nm}, \ell_{s} \cong 670 \mathrm{~nm}$ from Eq. (10), about an order of magnitude smaller than the size scale for the corresponding single crystals reported elsewhere [2].

\section{Length Scale Application to Thin Film Delamination}

If the volume to surface ratio concept is controlled by the balance of surface work and plastic energy dissipation, clearly it is applicable to thin film delamination which can follow the modified Griffith criterion. This is schematically shown in Figures 4(a) and (b) for indentation and film delamination. With initial indentation only plastic deformation in a zone of length $2 c$ forms as addressed above. This defines $\ell_{s}=V / S$. With the slightest increase of load this could trigger interface cracking at $2 b_{0}$ which then propagates and arrests at $2 b$. Now the plastic energy dissipation of importance to arrest is the deformation in the plane strain plastic zone at the advance of the crack front. We use the plane strain zone for two reasons here. First, we will apply this to films on rigid substrates where the plasticity is constrained. Second, a further constraint is provided by a superlayer of a high modulus material such as tungsten or tantalum nitride which has been applied on top of the ductile film to store elastic energy for the crack propagation process. This superlayer technique has been the subject of a number of recent investigations $[15,16]$. This constrained plane strain plastic zone size is given by

$$
R_{P_{I}}=\frac{K_{I}^{2}}{3 \pi \sigma_{y s}^{2}}
$$

where $K_{I}$ is the applied stress intensity factor. At this point the plastic volume for arrest can be assessed considering a plastic annulus of outer diameter $b+R_{P_{I}}$, inner diameter $b$ and height $R_{P_{I}}$, giving

$$
V=R_{P_{I}}\left[\pi\left[b+R_{P_{I}}\right]^{2}-\pi b^{2} R_{P_{I}}\right]=2 \pi\left(b R_{P_{I}}^{2}+R_{P_{I}}^{3} / 2\right) .
$$

The corresponding surface area created by this event is the film delamination given by

$$
S=\pi\left(b^{2}-b_{0}^{2}\right)=\pi\left[\left(b_{0}+\Delta b\right)^{2}-b_{0}^{2}\right] \cong 2 \pi \Delta b\left(b_{0}+\frac{\Delta b}{2}\right) .
$$

From (12) and (13) we see that the ratio is

$$
\frac{V}{S}=\frac{2 R_{P_{I}}^{2} b+R_{P_{I}}^{3}}{2 \Delta b b_{0}+\Delta b^{2}}
$$

For a number of trials using a sensible range for the incremental crack growth (blister size) and the plastic zone size we calculated $V / S$ from Eq. (14) to be

$$
\frac{25 R_{P_{I}}^{2}}{16 \Delta b}<\ell_{s}<\frac{10}{3} \frac{R_{P_{I}}^{2}}{\Delta b} \quad ; \quad 2 b_{0}<\left(\Delta b_{0}, R_{P_{I}}\right)<8 b_{0}
$$


which varies by about a factor of two. We're inclined toward the upper limit which represents crack-tip plastic zones smaller than the contact plastic zone. At this point we make two simplifying assumptions, one tenuous and one supported by observation. The first is that the length scale associated with plastic deformation during thin-film indentation is the same as that associated with producing crack-tip plasticity in thin films. Consider a typical thin film delamination fracture with $K_{I c} \cong 1 \mathrm{MPa} \cdot \mathrm{m}^{1 / 2}$ for a $300 \mathrm{~nm}$ thick film having a yield strength of 500 $\mathrm{MPa}$. This gives a plane strain plastic zone at the crack tip to be $420 \mathrm{~nm}$ from Eq. (11) and compares to the $1000 \mathrm{~nm}$ indentation plastic zone calculated from Eq. (8). Since the volume scale of these two zones is similar, we propose that the same length scale should apply. The second assumption, as supported by observations using focused ion-beam machining (FIB) is that the contact radius just prior to delamination, $a_{0}$, is the same as the initiation defect size, $b_{0}$.

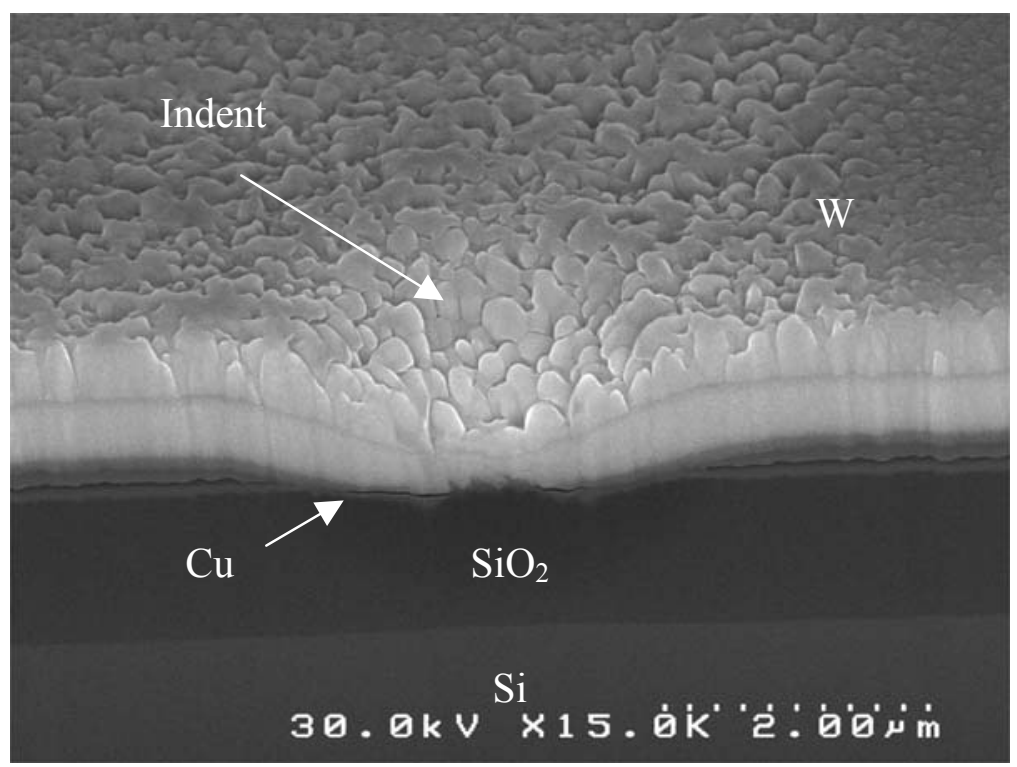

Figure 5: SEM image of the Focused Ion Beam cross-section of a delamination in $\mathrm{Cu}$ film.

As seen in Figure 5, a FIB cross-section of a copper film delaminated from a silicon wafer shows this to be reasonable. With $\ell_{s}^{I} \sim \ell_{s}^{c}$ being the same size scale for indentation (I) and cracking (c) of these films and $a \sim b_{0}$ one finds from (10), (11) and the approximate upper limit of (15) that

$$
\ell_{s}=\frac{\alpha h^{2}}{b_{0}} \cong \frac{3 R_{P_{I}}^{2}}{\Delta b} \cong \frac{K_{I}^{4}}{3 \pi^{2} \sigma_{y s}^{4} \Delta b} .
$$

From the first and fourth equalities this gives

$$
K_{I}=(\pi \sqrt{3})^{1 / 2} \sigma_{y s} \ell_{s}^{1 / 4} \Delta b^{1 / 4}
$$

From the second and fourth equalities, this reduces to

$$
K_{I} \cong \sigma_{y s}(\pi h)^{1 / 2}\left(\frac{3 \alpha \Delta b}{b_{0}}\right)^{1 / 4}
$$


Given that the strain energy release rate is $K_{I}^{2} / E$, we see that this represents a resistance curve of

$$
G_{R}=\frac{\pi \sigma_{y s}^{2} h}{E}\left(\frac{3 \alpha \Delta b}{b_{0}}\right)^{1 / 2}
$$

where $\Delta b / b_{0}$ is the ratio of incremental crack extension to the initial defect size and $\sigma_{y s}^{2} h / E$ is essentially the leading term in nearly all thin film resistance models involving plasticity. This proposed $R$-curve behavior for forced thin film crack extension is seen to evolve directly from a simple volume to surface length scale. To recapitulate, we propose that both indentation and fracture occurring from small volume deformation are controlled by the same length scale and that this leads to a delamination resistance criteria. Some verification of this proposed model based on existing thin film $\mathrm{Cu}$ data follows.

\section{EXPERIMENTAL}

A considerable amount of thin-film $\mathrm{Cu}$ delamination data exists as derived from superlayer indentation $[15,16]$. A schematic of this test is shown in Figure 6. This has shown that the thin film fracture energy increases with film thickness, a result consistent with 4-pt. bend data independently determined by Lane and Dauskardt [17]. An example of the blister formed by indenting a $1 \mu \mathrm{m}$ film of $\mathrm{W}$ bonded to a $\mathrm{Cu}$ film with a Ti underlayer between the $\mathrm{Cu}$ and the silicon substrate is shown in Figure 7.

a)

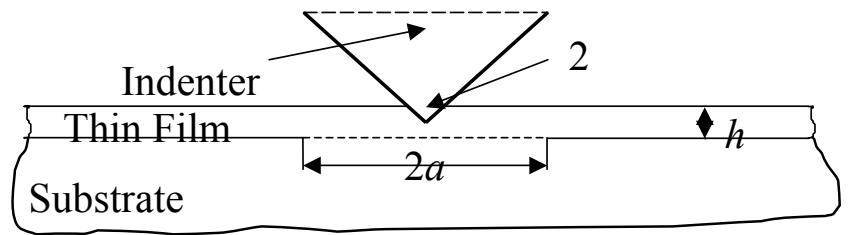

b)

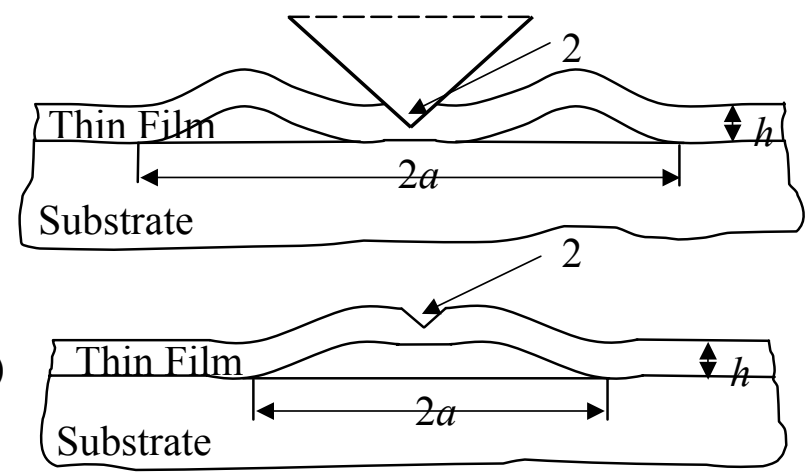

Figure 6: a) No buckling during indentation; b) double-buckling during indentation; c) singlebuckling after the indenter tip removal.

At the time we noted a typical factor of three scatter in adhesion energy which we originally attributed to a $b / b_{0}$ effect different that that represented by Eq. (17a). Consider then the case for the same film loaded repeatedly to ever increasing loads at different indentation locations as was accomplished here. Assume the crack starts in each case when the penetration reaches a critical 
contact radius, say $b_{0} \sim \mathrm{h}$, since then the spherical indentation nearly reaches the interface. It is easy to see that if the penetration continues that $\Delta b / b_{0}$ increases and the resistance must correspondingly increase to support larger stored elastic energy release rates at higher applied loads. This is different from a purely brittle interface where a crack, once triggered, will grow and then arrest with the larger the $b / b_{0}$ the lower the fracture resistance. This latter result is obtained from the driving force side of the equation as has been derived by Marshall and Evans [18] and Rosenfeld et al. [19]. The analysis associated with Eq. (17a) concerns itself with the resistance side of the equation and what happens if further crack extension along an elastic-plastic interface beyond the initial arrest is driven by ever increasing loads. Equation (17a) is consistent with the slow crack growth observations of $\mathrm{Au} / \mathrm{Al}_{2} \mathrm{O}_{3}$ interfaces [20] and remarkably similar to schematics

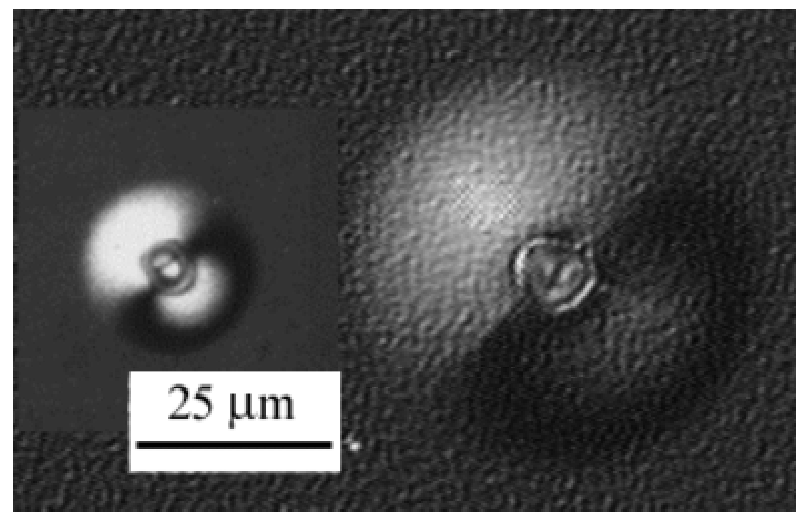

showing resistance curves ( $\Gamma_{R}$ vs. $\Delta a$ ) for models based upon the embedded process zone [21].

Figure 7: Optical micrographs of indentation iduced blisters with (right) and without (left) a W superlayer.

To ascertain if such a simple length scale model can predict crack growth resistance we reexamined some $\mathrm{Cu}$ thin film data based on the superlayer indentation technique. For $120 \mathrm{~nm}$ and $3.3 \mu \mathrm{m}$ thick $\mathrm{Cu}$, data are shown in Table 2. Referring to the schematic of Figure 4, we show the corresponding values of $b / b_{0}$, penetration depth, $\delta$, incremental crack extension, $\Delta b$, and the measured strain energy release rate from laminate composite analysis [7]. The latter is an extension of the Marshall and Evans analysis [18]. In the tabulation for both thicknesses, it is seen that as $\Delta b / b_{0}$ increases, $G_{I}$ generally increases. For a more quantitative comparison, it was necessary to obtain the length scale relationship as had been accomplished for aluminum films and described at Eq. (10). For Cu thicknesses of $200 \mathrm{~nm}$ to $2000 \mathrm{~nm}$ thick deposited on Si substrates with a TiW innerlayer, we have preliminary results. For these two thicknesses, we find that the form of Eq. (10) is similar with the only difference being the constant $\alpha \sim 4.8$. This is shown for one thickness in Figure 8. This gives

$$
G_{R}=\frac{12 \sigma_{y s}^{2} h}{E}\left(\frac{\Delta b}{b_{0}}\right)^{1 / 2}
$$

and is seen to correspond well to the data of Table 2 reproduced in Figure 9. Here, we have used $\sigma_{y s}=600 \mathrm{MPa}$ for the $120 \mathrm{~nm}$ film and $\sigma_{y s}=460 \mathrm{MPa}$ for the $3300 \mathrm{~nm}$ film. Equation (17b) slightly underpredicts the thicker films and overpredicts the thinner ones in Figure 9 implying that the factor of 16 difference in toughness predicted is too small. On the other hand, using a 4-pt. bend technique, Lane and Dauskardt observed only a factor of four increase in toughness for the same increase in thickness. Note that this difference in the experimental values could be real due 
to differences in bond strengths giving differences in $\Delta b / b_{0}$ for the same film. In these two sets of data, Volinsky, et al. $[15,16]$ used a Ti bond layer while Lane and Dauskardt [17] used a Ta/TaN

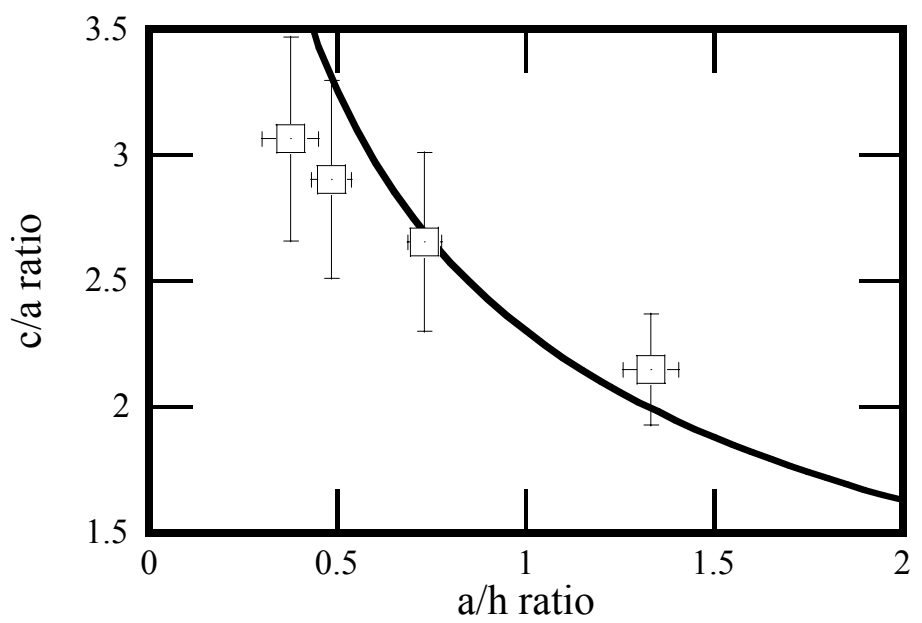

Figure 8: Fit of Eq. (8) to nanoindentation produced plastic zone radii, $c$, normalized on contact radii, $a$, as a function of $a / h$ with $h$ being film thickness. For these $\mathrm{Cu}$ films, $\alpha \cong 4.8$.

bond layer between the $\mathrm{Cu}$ and the substrate. Furthermore, there is a degree of uncertainty about $a_{0} \sim b_{0}$ which could easily account for any difference between prediction and observation. For these reasons we propose that this type of volume/surface approach be a serious candidate for the length scale controlling deformation and fraction of small volumes.

TABLE 2

\begin{tabular}{|c|c|c|c|c|}
\hline Cu Thickness, nm & $\begin{array}{c}\text { Depth, } \delta, \\
\text { nm }\end{array}$ & $\begin{array}{c}\text { Delamination } \\
\text { Radius, } b, \mathrm{~nm}\end{array}$ & $\left(\Delta b / b_{0}\right)^{1 / 2}$ & $G_{I}$, obs. \\
\hline \multirow{2}{*}{120} & 546 & 6800 & 1.67 & 2.2 \\
& 555 & 6800 & 1.67 & 2.3 \\
& 805 & 8400 & 1.91 & 4.5 \\
& 819 & 9000 & 2.00 & 3.7 \\
& 830 & 8300 & 1.90 & 5.6 \\
& 941 & 10,900 & 2.25 & 3.2 \\
& 952 & 11,200 & 2.29 & 2.9 \\
& 1067 & 1,900 & 2.37 & 3.9 \\
& 1083 & 11,700 & 2.39 & 5.9 \\
& 1116 & 22,300 & 2.35 & 11.9 \\
& 1549 & 21,200 & 3.42 & 9.4 \\
& 1869 & 31,900 & 4.09 & 8.95 \\
\hline 1892 & 22,300 & 3.37 & 59.9 \\
& 1910 & 21.900 & 3.34 & 57.3 \\
& 1915 & 6500 & 0.95 & 131.1 \\
& 1767 & 6700 & 0.985 & 101.3 \\
& 1783 & 6700 & 0.985 & 115.6 \\
& 1811 & 7700 & 1.12 & 123.7 \\
& 2344 & 8200 & 1.19 & 146.5 \\
& 2351 & 8200 & 1.19 & \\
& 2412 & 9900 & 1.38 & 1.35 \\
\hline
\end{tabular}




\section{SUMMARY}

We have first shown a connectivity between mesoscopic and macroscopic deformation theories through plastic pile-up around a nanoindenter. This recovers the classic dislocation pileup model of Eshelby [13]. To achieve this, a volume $(V)$ to surface $(S)$ ratio model has been invoked, previously shown $[2,6]$ to predict the indentation size effect (ISE) at small penetration depths. The length scale, $\ell_{s}=V / S$, has then been shown to apply to both small volume deformation and fracture through nanoindentation studies of thin film copper bonded to silicon substrates. This produces a fracture toughness which is proportional to the fourth root of the length scale or, alternatively, a resistance curve with the resistance proportional to the square root of the incremental crack growth. With increasing crack growth it is shown that $\mathrm{Cu}$ bonded to silicon substrates with a thin Ti layer increases its fracture resistance by a factor of three.

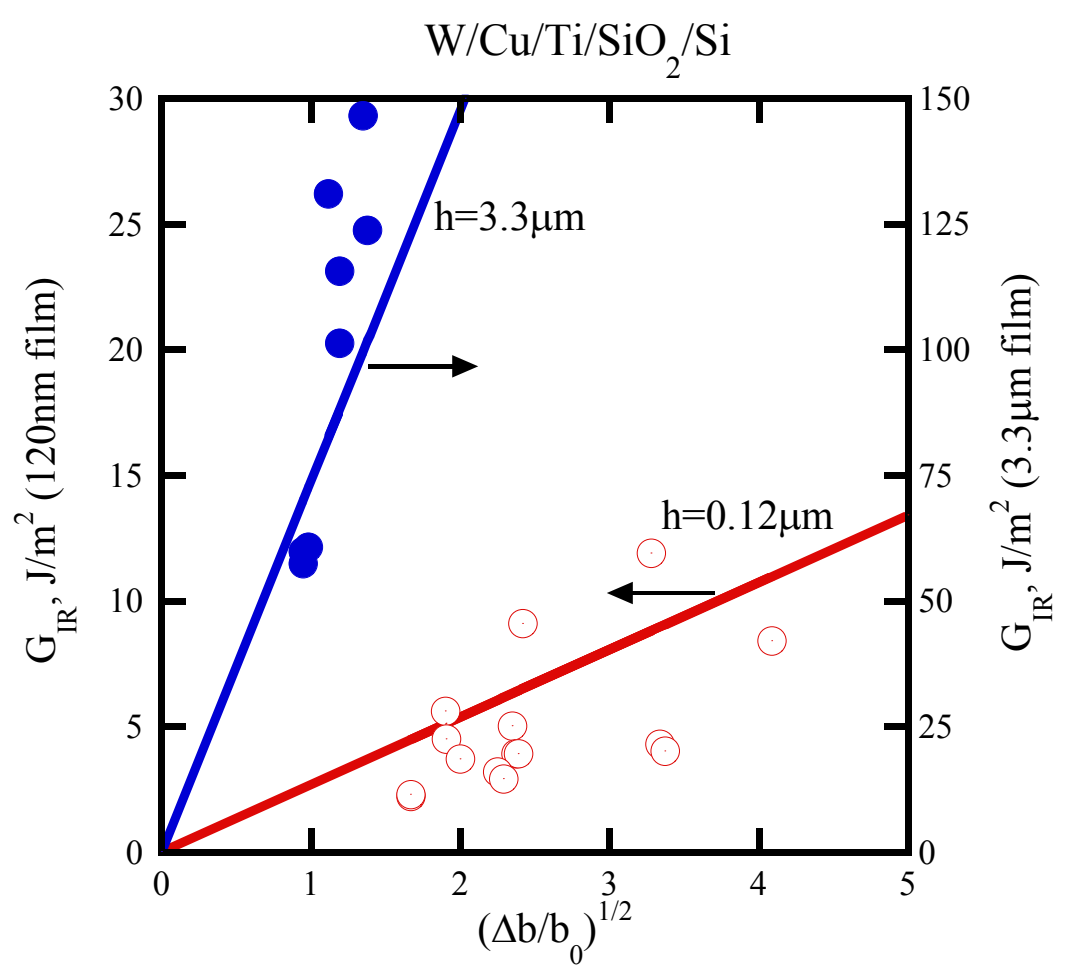

Figure 9: Crack growth resistance of $\mathrm{Cu}$ films as a function of incremental crack extension compared to Eq. (17b).

\section{ACKNOWLEDGMENTS}

The authors would like to acknowledge support through MINT (The Center for Micromagnetics and Information Technology) via Seagate Technology, Motorola, Inc., and the Department of Energy under DOE contract DE-FG02/96ER45574. We would also like to thank Indira S. Adhihetty, Joseph B. Vella, Robert H. Caprenter and Lester Casoose Jr. of Motorola, Inc. for help with the FIB analysis. 


\section{REFERENCES}

1. Horstemeyer, M.E. and Baskes, M.I. (1999) J. Engng. Mater. Tech., Trans. ASME 121, 114.

2. Gerberich, W.W., Tymiak, N.I., Grunlan, J.C., Horstemeyer, M.F. and Baskes, M.I. (2001) "Interpretations of Indentation Size Effects" J. Appl. Mech. submitted.

3. $\quad$ Bagchi, A., Lucas, G., Suo, Z. and Evans, A. (1994) J. Mater. Res. 9(7), 1734.

4. Vlassak, J.J., Drory, M.D. and Nix, W.D. (1997) J. Mater. Res. 12, 1900.

5. Begley, M.R., Mumm, D.R., Evans, A.G. and Hutchinson, J.W. (2000) Acta Mater. 48, 3211.

6. Tymiak, N.I., Kramer, D.E., Bahr, D.F., Wyrobek, T.J. and Gerberich, W.W. (2001) Acta Mater. 49, 1021.

7. Kriese, M.D., Moody, N.R. and Gerberich, W.W. (1999) J. Mater. Res. 14(7), 3007 (Part I); 3019 (Part II).

8. Volinksy, A.A., Tymiak, N.I., Kriese, M.D., Gerberich, W.W. and Hutchinson, J.W. (1999) Mater. Res. Soc. Proc. 539, 277.

9. Gerberich, W.W., Lilleodden, E.T., Foecke, T.J. and Wyrobek, T.J. (1995). In Micromechanics of Advanced Materials, pp. 29-35, J.C.M. Li Symposium, TMS, Warrendale, PA.

10. Gerberich, W.W., Tymiak, N.I., Kramer, D.E., Daugela, A., Jungk, J. and Li, M. (2001) "An Approach to Dry Friction and Wear for Small Volumes" Philos. Mag. A, submitted.

11. Harvey, S., Huang, H., Venkataraman, S. and Gerberich, W.W., (1993) J. Mater. Res. 8(6), 1291.

12. Johnson, K.L. (1970) J. Mech. Phys. Solids 18, 115.

13. Eshelby, J.D., Frank, F.C. and Nabarro, F.R.N. (1951) Philos. Mag. 42, 351.

14. Kramer, D.E., Volinsky, A.A., Moody, N.R. and Gerberich, W.W. (2000) "Substrate Effects on Indentation Plastic Zone Development in Thin Soft Films," J. Mater. Res., accepted.

15. Tymiak, N.I., Volinksy, A.A., Kriese, M.D., Downs, S.A. and Gerberich, W.W. (1999) Metall. Mater. Trans. 31A, 863.

16. Volinksy, A.A., Moody, N.R. and Gerberich, W.W. (2001) "Interfacial Toughness Measurements of Thin Metal Films," Acta Mater., submitted.

17. Lane, M. and Dauskardt, R.H. (2000) J. Mater. Res. 15(1), 203.

18. Marshall, D.B. and Evans, A.G. (1984) J. Appl. Phys. 56, 2632.

19. Rosenfeld, L.G., Ritter, J.E., Lardner, T.J. and Lin, M.R. (1990) J. Appl. Phys. 67(7), 3291.

20. Lipkin, D.M., Clarke, D.R. and Evans, A.G. (1998) Acta Mater.47(13), 4835.

21. Evans, A.G., Hutchinson, J.W. and Wei, Y. (1999) Acta Mater. 47(15), 4093. 